\title{
O conhecimento dos discentes de enfermagem acerca do câncer de colo do útero
}

\author{
The knowledge of nursing students about cervical cancer
}

El conocimiento de los estudiantes de enfermería sobre el cáncer de cuello uterino

\section{José Antonio da Silva Júnior ${ }^{I}$, Luana Larissa Oliveira Bezerra ${ }^{\mathrm{II}}$, Javanna Lacerda Gomes da Silva Freitas $^{\mathrm{III}}$, Sheila Milena Pessoa dos Santos ${ }^{\mathrm{IV}}$, Rodrigo Pinheiro Fernandes de Queiroga ${ }^{\mathrm{V}}$, Trycia Ryane de Freitas Silva ${ }^{\mathrm{VI}}$}

\begin{abstract}
Resumo: Objetivo: analisar o conhecimento dos discentes do curso de enfermagem acerca dos fatores de risco e prevenção do câncer de colo do útero. Método: estudo transversal, quantitativo, realizado com 112 discentes do curso de enfermagem de uma instituição pública de ensino superior no Nordeste do Brasil. Dados coletados em junho de 2017, utilizando questionário estruturado, com análise bivariada entre conhecimento sobre os fatores de risco e prevenção do câncer de colo do útero e as variáveis sociodemográficas, comportamentais e acadêmicas. Resultados: verificou-se associação entre o conhecimento acerca dos fatores de risco e prevenção e a participação dos discentes em atividades extracurriculares, período de curso e assistência à mulher com ênfase no câncer de colo do útero. Conclusão: salienta-se a necessidade de aprimoramento das abordagens dessa temática no curso de graduação em Enfermagem por meio da participação dos alunos em atividades extracurriculares.
\end{abstract}

Descritores: Educação em Enfermagem; Colo de Útero; Fatores de Risco; Prevenção de Doenças; Enfermagem

Abstract: Objective: to analyze the knowledge of nursing undergraduate students about the risk factors and prevention of cervical cancer. Method: a cross-sectional, quantitative study, carried out with 112 nursing students from a public higher education institution in Northeast Brazil. Data collected in June 2017, using a structured questionnaire, with bivariate analysis between knowledge about the risk factors and prevention of cervical cancer and the sociodemographic, behavioral and academic variables. Results: there was an association between the undergraduate students' knowledge about risk and prevention factors and their participation in extracurricular activities, semester and assistance to women with an emphasis on cervical cancer. Conclusion: It is emphasized the

\footnotetext{
I Enfermeiro. Graduado em Enfermagem. Universidade Federal de Campina Grande, Campina Grande, Paraíba, Brasil. E-mail: joseantonio.030@hotmail.com; ORCID: https://orcid.org/0000-0001-7965-3095.

II Enfermeira. Graduada em Enfermagem. Universidade Federal de Campina Grande, Campina Grande, Paraíba, Brasil. E-mail: luana.olibe@gmail.com; ORCID: https://orcid.org/0000-0002-6661-9290.

III Enfermeira. Mestre em Saúde Pública. Universidade Federal de Campina Grande, Campina Grande, Paraíba, Brasil. E-mail: javanna.lacerda@hotmail.com; ORCID: https://orcid.org/0000-0003-4524-583X.

IV Enfermeira. Doutora em Enfermagem. Professora adjunta, Universidade Federal de Campina Grande, Campina Grande, Paraíba, Brasil. Email: sheila.milena@gmail.com; ORCID: http://orcid.org/0000-0001-9396-9192.

V Enfermeiro. Doutor em Enfermagem. Professor, Universidade Federal de Campina Grande, Campina Grande, Paraíba, Brasil. E-mail: rodrigopfq@gmail.com; ORCID: https://orcid.org/0000-0002-0642-6382.

VI Enfermeira. Graduada em Enfermagem. Universidade Federal de Campina Grande, Campina Grande, Paraíba, Brasil. E-mail: trycia_ryane@hotmail.com; ORCID: https://orcid.org/0000-0002-9568-362X.
} 
need to improve the approaches to this theme in the undergraduate nursing course through the participation of students in extracurricular activities.

Descriptors: Education, Nursing; Cervix Uteri; Risk Factors; Disease Prevention; Nursing

Resumen: Objetivo: analizar el conocimiento de los estudiantes de enfermería sobre los factores de riesgo y prevención del cáncer de cuello uterino. Método: estudio transversal, cuantitativo, realizado con 112 estudiantes de enfermería de una institución pública de educación superior en el noreste de Brasil. Los datos fueron recogidos en junio de 2017, a través de un cuestionario estructurado, con análisis bivariado entre el conocimiento sobre los factores de riesgo y prevención del cáncer de cérvix y las variables sociodemográficas, comportamentales y académicas. Resultados: comprobose asociación entre el conocimiento sobre factores de riesgo y prevención y la participación de los estudiantes en actividades extracurriculares, período de curso y asistencia a mujeres con énfasis en cáncer de cuello uterino. Conclusión: se enfatiza la necesidad de mejorar los enfoques de esta temática en el grado de enfermería a través de la participación de los estudiantes en las actividades extracurriculares.

Descriptores: Educación en Enfermería; Cuello del Útero; Factores de Riego; Prevención de Enfermedades; Enfermería

\section{Introdução}

O câncer de colo do útero (CCU) é uma das principais causas de morte entre as mulheres do mundo. ${ }^{1}$ Em 2018, essa doença causou 311 mil mortes, principalmente em países de baixa e média renda. ${ }^{2}$ No Brasil, esse câncer ocupa a terceira posição dentre os tumores mais incidentes em mulheres, sendo estimado que para cada ano do triênio 2020/2022 a incidência seja de 16.590 casos desse câncer, com um risco estimando de 15,43 casos a cada 100 mil mulheres. ${ }^{3}$

O principal fator de risco para o desenvolvimento do CCU é a infecção pelo Human Papiloma Virus (HPV), presente em $99,7 \%$ dos casos. ${ }^{4}$ Outros fatores que podem influenciar na regressão ou na evolução da infecção são: imunossupressão, tabagismo, paridade, coinfecções sexualmente transmitidas e fatores comportamentais, os quais podem ser prevenidos, por isso são alvos de estratégias de ações de prevenção primária e secundária. ${ }^{14-5}$

A prevenção primária está relacionada à diminuição do risco de contágio com o HPV. Dessa forma, são incluídas como ações em saúde: a vacinação de adolescentes contra o citado vírus, a utilização de preservativos em todas as relações sexuais e as ações de educação em saúde para a população frisando os fatores de risco. ${ }^{1}$ 
A prevenção secundária, por sua vez, inclui ações de diagnóstico precoce por intermédio da identificação, em tempo oportuno, dos sinais e sintomas relacionados ao CCU, como a presença de sangramentos intermenstruais, de corrimento sanguinolento, de dor pélvica e da detecção de lesões precursoras por meio do rastreamento, utilizando a citopatologia oncótica de mulheres entre 25 e 64 anos com vida sexual ativa. ${ }^{1,4,6}$

Para o desenvolvimento das ações de prevenção do CCU, é necessário que os profissionais de saúde conheçam os principais fatores de risco e desenvolvam ações que garantam a promoção da saúde, o diagnóstico precoce e o tratamento eficaz. ${ }^{7}$ Logo, é fundamental que os enfermeiros estejam aptos e capacitados para realização de uma abordagem apropriada para essas mulheres, em consonância com as diretrizes para prevenção. ${ }^{8}$ Para tanto, o enfermeiro deve ser capaz de atender às mulheres de forma integral, efetuar consultas de enfermagem e exame clínico, avaliar resultados de exames laboratoriais, realizar cuidados paliativos e desenvolver atividades de educação permanente para todos os membros da equipe. ${ }^{4,9}$

Tendo em vista o papel do enfermeiro diante das ações de prevenção do CCU, destaca-se a sua formação profissional generalista e focada na humanização e na educação em saúde, ${ }^{10}$ tornando-os capazes de contribuir com a promoção da redução dos fatores de risco para o CCU e com o fortalecimento de ações de prevenção primária e secundária.

No âmbito da formação profissional, ressalta-se o fato de que, segundo estudos internacionais, ${ }^{11-12}$ o conhecimento dos discentes de enfermagem ainda é insuficiente acerca da prevenção do CCU. Tal fato é preocupante, tendo em vista que o enfermeiro, desde a graduação, precisa se autoavaliar e refletir sobre o seu papel enquanto profissional da saúde, a fim de elaborar planos de cuidados baseados nas necessidades da população. ${ }^{13}$ Portanto, destaca-se a relevância da promoção de vivências práticas dos discentes durante a graduação, objetivando aproximação com o seu papel enquanto profissional da saúde. ${ }^{14}$ 
O conhecimento dos discentes de enfermagem acerca do câncer de colo do útero | 4

Nesse contexto, torna-se pertinente a temática desta pesquisa, pois os estudos citados mencionam que os discentes não possuem conhecimento suficiente acerca das formas de prevenção do CCU, que a formação acadêmica do enfermeiro é alicerce para a sua prática profissional e que o enfermeiro tem papel fundamental nas ações de promoção e proteção das mulheres. Salienta-se que, na literatura consultada, poucos estudos analisaram a interface da formação em enfermagem e os fatores de risco e prevenção do referido tipo de câncer, especialmente quando se trata dos conhecimentos específicos adquiridos durante a graduação sobre esse tema. ${ }^{15-17}$

Diante desse cenário, questionou-se: qual o conhecimento dos discentes de enfermagem acerca dos fatores de risco e prevenção primária e secundária do CCU? Desse modo, este estudo objetivou analisar o conhecimento dos discentes do curso de enfermagem acerca dos fatores de risco e prevenção do câncer de colo do útero.

\section{Método}

Trata-se de um estudo transversal, de abordagem quantitativa, produzido a partir de um recorte de pesquisa de iniciação científica, a qual teve como título “Atenção à saúde com foco no câncer cervical: conhecimento de discentes de enfermagem”. A população foi composta por discentes do curso de graduação em enfermagem de uma Universidade Federal no Nordeste do Brasil. Foram incluídos todos os discentes matriculados no curso, de ambos os sexos, com idade igual ou superior a 18 anos. Foram excluídos os alunos que haviam faltado às aulas nos dias da coleta dos dados, além dos que estavam participando da elaboração e organização deste estudo, totalizando 112 discentes participantes.

A coleta de dados foi realizada no mês de junho de 2017, nas respectivas salas de aula, de acordo com o período de curso do discente. Utilizou-se um questionário estruturado, elaborado 
5 | Silva Júnior JA, Bezerra LLO, Freitas JLGS, Santos SMP, Queiroga RPF, Silva TRF

com base em guias e diretrizes para o controle do CCU e composto pelos eixos: informações pessoais, informações acadêmicas, conhecimentos gerais e específicos sobre CCU.1,4,6

A variável dependente foi o conhecimento acerca do CCU, avaliada em três perspectivas: fatores de risco, prevenção primária e prevenção secundária do CCU. ${ }^{4}$ As variáveis independentes foram divididas em: 1) sociodemográficas e comportamentais: sexo (feminino; masculino), faixa etária (18-23; $>23$ anos), início da atividade sexual (sim; não), vida sexual ativa (sim; não); e 2) acadêmicas: participação em atividades extracurriculares durante a graduação (sim; não), período do curso (primeira metade; segunda metade) e assistência à mulher durante a graduação, com ênfase no CCU (sim; não).

Em relação à variável “participação em atividades extracurriculares durante a graduação”, foram consideradas as atividades de monitoria em componentes curriculares da graduação, o Programa de Educação pelo Trabalho para a Saúde (PET-Saúde), a participação em ligas acadêmicas, em projetos de extensão e de iniciação científica.

A variável "período do curso" foi categorizada em primeira metade e segunda metade do curso, incluindo nessas categorias os discentes do primeiro ao quarto período e do quinto ao décimo período, respectivamente. Tendo em vista que o componente curricular Saúde da Mulher na instituição de ensino superior em que o estudo foi desenvolvido é ministrado no quinto período, categorizou-se essa variável, visando classificar os discentes como os que não contactaram com a temática (primeira metade do curso) e os que contactaram com a temática (segunda metade do curso).

No tocante à "assistência à mulher com ênfase no CCU”, foram avaliados os discentes que haviam vivenciado ou não práticas de assistência direta à saúde da mulher durante a graduação, por meio da realização de consultas de enfermagem para esse público específico, educação em saúde e ações em projetos. 
O conhecimento dos discentes de enfermagem acerca do câncer de colo do útero |6

Para tabulação e análise dos dados, utilizou-se o software estatístico Epi Info ${ }^{T M}$ versão 7.2.2.2. Os dados foram tabulados em dupla entrada e foram organizados em tabelas com as frequências absolutas e relativas. Para a análise bivariada, utilizou-se o teste de qui-quadrado de Pearson, sendo utilizado o teste exato de Fisher para resultados com contagem esperada menor que cinco, com nível de significância $\alpha=0,05$. A discussão dos dados foi realizada à luz da literatura específica sobre o CCU.

A pesquisa foi aprovada no dia 30 de maio de 2017 pelo Comitê de Ética em Pesquisa do Hospital Universitário Alcides Carneiro, sob parecer de número 2.091.521 e CAAE nº 68682017.5.0000.5182. Após a aprovação do projeto, os discentes expressaram vontade em participar deste estudo por meio da assinatura do Termo de Consentimento Livre e Esclarecido.

Cabe salientar que em todo o processo da pesquisa e construção deste artigo foram respeitados os preceitos contidos na Resolução 466/2012 do Conselho Nacional de Saúde. Os resultados deste estudo foram devolvidos aos discentes por meio de palestras e orientações acerca da temática a partir das fragilidades identificadas.

\section{Resultados}

Participaram deste estudo 112 discentes, dos quais as maiores proporções foram: sexo feminino (76,8\%), faixa etária entre 18 e 23 anos (76,8\%), discentes que iniciaram a vida sexual $(69,4 \%)$ e que possuíam vida sexual ativa $(55,4 \%)$. Quanto à formação acadêmica, a maioria pertencia a segunda metade do curso (62,5\%), havia participado de alguma atividade extracurricular $(75,9 \%)$ e havia prestado assistência à mulher com ênfase no CCU $(51,8 \%)$.

Não houve associação estatística entre as variáveis sociodemográficas e comportamentais (sexo, faixa etária, início da atividade sexual, vida sexual ativa) e o conhecimento específico acerca dos fatores de risco e as formas de prevenção primária e secundária do CCU. Contudo, 
7 | Silva Júnior JA, Bezerra LLO, Freitas JLGS, Santos SMP, Queiroga RPF, Silva TRF

observou-se associação estatística significativa entre o conhecimento voltado aos fatores de risco e as variáveis acadêmicas dos discentes, a qual está disposta nas Tabelas 1 e 2.

Tabela 1 - Associação entre o conhecimento acerca da infecção por HPV e do tabagismo e as variáveis acadêmicas dos discentes. Campina Grande, Paraíba, Brasil, 2017.

\begin{tabular}{|c|c|c|c|c|c|c|c|c|c|c|}
\hline \multirow{3}{*}{ Variáveis } & \multicolumn{4}{|c|}{ Infecção por HPV } & \multicolumn{6}{|c|}{ Tabagismo } \\
\hline & \multicolumn{2}{|c|}{ Certo } & \multicolumn{2}{|c|}{ Errado } & \multirow{2}{*}{$\mathrm{p}$} & \multicolumn{2}{|c|}{ Certo } & \multicolumn{2}{|c|}{ Errado } & \multirow[b]{2}{*}{$\mathrm{p}$} \\
\hline & n & $\%$ & $\mathbf{n}$ & $\%$ & & $\mathbf{n}$ & $\%$ & $\mathbf{n}$ & $\%$ & \\
\hline \multicolumn{11}{|c|}{ Participação de atividades extracurriculares } \\
\hline $\operatorname{Sim}$ & 80 & 94,1 & 05 & 5,9 & \multirow{2}{*}{$1,000^{*}$} & 20 & 23,5 & 65 & 76,5 & \multirow{2}{*}{0,586} \\
\hline Não & 26 & 96,3 & 01 & 3,7 & & 05 & 18,5 & 22 & 81,5 & \\
\hline \multicolumn{11}{|l|}{ Período do curso } \\
\hline Primeira metade & 36 & 85,7 & 06 & 14,3 & \multirow{2}{*}{$0,002^{*}$} & 09 & 21,4 & 33 & 78,6 & \multirow{2}{*}{0,860} \\
\hline Segunda metade & 70 & 100 & - & -- & & 16 & 22,9 & 54 & 77,1 & \\
\hline \multicolumn{11}{|c|}{ Assistência à mulher com ênfase no CCU } \\
\hline Assistiu & 58 & 100 & - & -- & \multirow{2}{*}{$0,001^{*}$} & 12 & 20,7 & 46 & 79,3 & \multirow{2}{*}{0,667} \\
\hline Nunca assistiu & 48 & 88,9 & 06 & 11,1 & & 13 & 24,1 & 41 & 75,9 & \\
\hline
\end{tabular}

${ }^{*}$ Teste exato de Fisher.

Tabela 2 - Associação entre o conhecimento sobre a multiplicidade de parcerias sexuais e do início precoce da vida sexual e as variáveis acadêmicas dos discentes. Campina Grande, Paraíba, Brasil, 2017.

\begin{tabular}{|c|c|c|c|c|c|c|c|c|c|c|}
\hline \multirow{3}{*}{ Variáveis } & \multicolumn{5}{|c|}{$\begin{array}{c}\text { Multiplicidade } \\
\text { de parcerias }\end{array}$} & \multicolumn{5}{|c|}{ Início precoce da vida sexual } \\
\hline & \multicolumn{2}{|c|}{ Certo } & \multicolumn{2}{|c|}{ Errado } & \multirow{2}{*}{$\mathrm{p}$} & \multicolumn{2}{|c|}{ Certo } & \multicolumn{2}{|c|}{ Errado } & \multirow{2}{*}{$\mathrm{p}$} \\
\hline & n & $\%$ & $\mathrm{n}$ & $\%$ & & $\mathbf{n}$ & $\%$ & $\mathbf{n}$ & $\%$ & \\
\hline \multicolumn{11}{|c|}{ Participação de atividades extracurriculares } \\
\hline Sim & 63 & 74,1 & 22 & 25,9 & \multirow{2}{*}{0,004} & 25 & 29,4 & 60 & 70,6 & \multirow{2}{*}{0,070} \\
\hline Não & 12 & 44,4 & 15 & 55,6 & & 03 & 11,1 & 24 & 88,9 & \\
\hline \multicolumn{11}{|l|}{ Período do curso } \\
\hline Primeira metade & 19 & 45,2 & 23 & 54,8 & \multirow{2}{*}{$<0,001$} & 05 & 11,9 & 37 & 88,1 & \multirow{2}{*}{0,019} \\
\hline Segunda metade & 56 & 80,0 & 14 & 20,0 & & 22 & 31,4 & 48 & 68,6 & \\
\hline \multicolumn{11}{|c|}{ Assistência à mulher com ênfase no CCU } \\
\hline Assistiu & 45 & 77,6 & 13 & 22,4 & \multirow{2}{*}{0,013} & 15 & 25,9 & 43 & 74,1 & \multirow{2}{*}{0,653} \\
\hline Nunca assistiu & 30 & 55,6 & 24 & 44,4 & & 12 & 22,2 & 42 & 77,8 & \\
\hline
\end{tabular}

Em relação ao conhecimento a respeito da infecção pelo HPV como fator de risco para o CCU, identificou-se associação estatística com o período do curso $(p=0,002)$ e com a assistência à mulher com ênfase no CCU $(p=0,011)$. Quanto ao tabagismo, não houve associação estatística com as variáveis analisadas. No que se refere à multiplicidade de parcerias sexuais, verificou-se 
a mesma associação com a participação em atividades extracurriculares $(\mathrm{p}=0,004)$, período do curso $(\mathrm{p}<0,001)$ e assistência à mulher com ênfase no CCU $(\mathrm{p}=0,013)$.

Referente ao início precoce da vida sexual como fator de risco, observou-se que houve associação estatística significativa com o período do curso $(\mathrm{p}=0,019)$. Apesar da associação significativa para essa variável e do número de acertos entre os discentes da segunda metade do curso ter sido relativamente maior do que aqueles da primeira metade, percebeu-se que a proporção de acertos foi baixa $(31,4 \%)$, tendo em vista o conhecimento prévio em relação à essa temática no citado grupo de discentes.

A associação estatística entre o conhecimento relacionado à prevenção primária do CCU e as variáveis acadêmicas dos discentes encontra-se disposta na Tabela 3.

Tabela 3 - Associação entre o conhecimento acerca da prevenção primária do CCU e as variáveis acadêmicas dos discentes. Campina Grande, Paraíba, Brasil, 2017

\begin{tabular}{|c|c|c|c|c|c|c|c|c|c|c|}
\hline \multirow{3}{*}{ Variáveis } & \multicolumn{4}{|c|}{ Uso de preservativos } & \multicolumn{6}{|c|}{ Vacina contra o HPV } \\
\hline & \multicolumn{2}{|c|}{ Certo } & \multicolumn{2}{|c|}{ Errado } & \multirow{2}{*}{$\mathrm{p}$} & \multicolumn{2}{|c|}{ Certo } & \multicolumn{2}{|c|}{ Errado } & \multirow{2}{*}{$\mathrm{p}$} \\
\hline & $\mathbf{n}$ & $\%$ & n & $\%$ & & $\mathrm{n}$ & $\%$ & $\mathbf{n}$ & $\%$ & \\
\hline \multicolumn{11}{|c|}{ Participação em atividades extracurriculares } \\
\hline $\operatorname{Sim}$ & 69 & 81,2 & 16 & 18,8 & \multirow{2}{*}{0,699} & 76 & 89,4 & 09 & 10,6 & \multirow{2}{*}{$0,190^{*}$} \\
\hline Não & 21 & 77,8 & 06 & 22,2 & & 21 & 77,8 & 06 & 22,2 & \\
\hline \multicolumn{11}{|l|}{ Período do curso } \\
\hline Primeira metade & 29 & 69,0 & 13 & 31,0 & \multirow{2}{*}{0,020} & 34 & 81,0 & 08 & 19,0 & \multirow{2}{*}{0,173} \\
\hline Segunda metade & 61 & 87,1 & 09 & 12,9 & & 63 & 90,0 & 07 & 10,0 & \\
\hline \multicolumn{11}{|c|}{ Assistência à mulher com ênfase no CCU } \\
\hline Assistiu & 52 & 89,7 & 06 & 10,3 & \multirow{2}{*}{0,010} & 54 & 93,1 & 04 & 6,9 & \multirow{2}{*}{0,036} \\
\hline Nunca assistiu & 38 & 70,4 & 16 & 29,6 & & 43 & 79,6 & 11 & 20,4 & \\
\hline
\end{tabular}

*Teste exato de Fisher.

No que concerne ao conhecimento dos discentes na perspectiva da prevenção primária para o CCU, verificou-se associação estatística entre o conhecimento do uso de preservativos e o período do curso $(\mathrm{p}=0,020)$; e a assistência à mulher com ênfase no CCU $(\mathrm{p}=0,010)$. A respeito da vacinação contra o HPV como forma de prevenção primária para o CCU, viu-se associação estatística com a assistência à mulher com ênfase no CCU ( $\mathrm{p}=0,036)$. Não foi identificada associação com o período do curso $(\mathrm{p}=0,173)$, nem com a participação em atividades 
extracurriculares $(\mathrm{p}=0,190)$. Observou-se que os discentes da primeira metade $(81,0 \%)$ e os da segunda metade $(90,0 \%)$ do curso possuíam conhecimento considerável acerca da temática.

Nas Tabelas 4 e 5 encontram-se os valores referentes à associação entre o conhecimento pautado na prevenção secundária do CCU e as variáveis acadêmicas dos discentes.

Tabela 4 - Associação entre o conhecimento direcionado ao rastreio e ao sangramento intermenstrual e as variáveis acadêmicas dos discentes. Campina Grande, Paraíba, Brasil, 2017.

\begin{tabular}{|c|c|c|c|c|c|c|c|c|c|c|}
\hline \multirow{3}{*}{ Variáveis } & \multicolumn{4}{|c|}{ Rastreio } & & \multicolumn{5}{|c|}{ Sangramento intermenstrual } \\
\hline & \multicolumn{2}{|c|}{ Certo } & \multicolumn{2}{|c|}{ Errado } & \multirow{2}{*}{$\mathrm{p}$} & \multicolumn{2}{|c|}{ Certo } & \multicolumn{2}{|c|}{ Errado } & \multirow{2}{*}{$\mathrm{p}$} \\
\hline & $\mathbf{n}$ & $\%$ & $\mathbf{n}$ & $\%$ & & $\mathbf{n}$ & $\%$ & $\mathbf{n}$ & $\%$ & \\
\hline \multicolumn{11}{|c|}{ Participação em atividades extracurriculares } \\
\hline Sim & 15 & 17,6 & 70 & 82,4 & \multirow{2}{*}{$0,237^{*}$} & 42 & 49,4 & 43 & 50,6 & \multirow{2}{*}{0,032} \\
\hline Não & 02 & 7,4 & 25 & 92,6 & & 07 & 25,9 & 20 & 74,1 & \\
\hline \multicolumn{11}{|l|}{ Período do curso } \\
\hline Primeira metade & 03 & 7,1 & 39 & 92,9 & \multirow{2}{*}{0,066} & 15 & 35,7 & 27 & 64,3 & \multirow{2}{*}{0,184} \\
\hline Segunda metade & 14 & 20,0 & 56 & 80,0 & & 34 & 48,6 & 36 & 51,4 & \\
\hline \multicolumn{11}{|c|}{ Assistência à mulher com ênfase no CCU } \\
\hline Assistiu & 12 & 20,7 & 46 & 79,3 & \multirow{2}{*}{0,092} & 29 & 50,0 & 29 & 50,0 & \multirow{2}{*}{0,167} \\
\hline Não assistiu & 05 & 9,3 & 49 & 90,7 & & 20 & 37,0 & 34 & 63,0 & \\
\hline
\end{tabular}

${ }^{*}$ Teste exato de Fisher.

Tabela 5 - Associação entre o conhecimento sobre o corrimento sanguinolento e a dor pélvica e as variáveis acadêmicas dos discentes. Campina Grande, Paraíba, Brasil, 2017.

\begin{tabular}{|c|c|c|c|c|c|c|c|c|c|c|}
\hline \multirow[t]{3}{*}{ Variáveis } & \multicolumn{5}{|c|}{ Corrimento sanguinolento } & \multicolumn{4}{|c|}{ Dor pélvica } & \multirow{3}{*}{$\mathrm{p}$} \\
\hline & \multicolumn{2}{|c|}{ Certo } & \multicolumn{2}{|c|}{ Errado } & \multirow[b]{2}{*}{$\mathrm{p}$} & \multicolumn{2}{|c|}{ Certo } & \multicolumn{2}{|c|}{ Errado } & \\
\hline & $\mathbf{n}$ & $\%$ & $\mathrm{n}$ & $\%$ & & $\mathbf{n}$ & $\%$ & $\mathbf{n}$ & $\%$ & \\
\hline \multicolumn{11}{|c|}{ Participação em atividades extracurriculares } \\
\hline $\operatorname{Sim}$ & 51 & 60,0 & 34 & 40,0 & \multirow{2}{*}{0,041} & 56 & 65,9 & 29 & 34,1 & \multirow{2}{*}{0,190} \\
\hline Não & 22 & 81,5 & 05 & 18,5 & & 14 & 51,9 & 13 & 48,1 & \\
\hline \multicolumn{11}{|l|}{ Período do curso } \\
\hline Primeira metade & 33 & 78,6 & 09 & 21,4 & \multirow{2}{*}{0,021} & 26 & 61,9 & 16 & 38,1 & \multirow{2}{*}{0,920} \\
\hline Segunda metade & 40 & 57,1 & 30 & 42,9 & & 44 & 62,9 & 26 & 37,1 & \\
\hline \multicolumn{11}{|c|}{ Assistência à mulher com ênfase no CCU } \\
\hline Assistiu & 33 & 56,9 & 25 & 43,1 & \multirow{2}{*}{0,057} & 39 & 67,2 & 19 & 32,3 & \multirow{2}{*}{0,283} \\
\hline Não assistiu & 40 & 74,1 & 14 & 25,9 & & 31 & 57,4 & 23 & 42,6 & \\
\hline
\end{tabular}

Acerca do rastreio como uma forma de prevenção secundária, não foi identificada associação estatística com nenhuma das variáveis analisadas. De modo geral, os participantes com experiência em atividades extracurriculares $(82,4 \%)$, que estavam na segunda metade do 
curso (80,0\%) e que haviam assistido alguma mulher com ênfase no CCU (79,3\%), não consideraram o rastreamento como uma forma de prevenção secundária do CCU.

Sobre o diagnóstico precoce do CCU por meio da sintomatologia característica, foi identificada associação significativa com a participação em atividades extracurriculares $(p=0,032)$. Em relação à presença do corrimento sanguinolento como sinal sugestivo de CCU, observou-se associação significativa com o período do curso $(\mathrm{p}=0,021)$ e com a participação em atividades extracurriculares $(\mathrm{p}=0,041)$. No que tange ao período do curso, identificou-se que houve maior taxa de acerto dos discentes da primeira metade do curso (78,6\%), quando comparados à segunda metade (57,1\%). Não houve associação estatística entre dor pélvica como sintomatologia sugestiva de diagnóstico precoce do CCU em nenhuma das variáveis analisadas.

\section{Discussão}

Embora o CCU seja o quarto tipo de câncer mais incidente no mundo e o terceiro no Brasil, ${ }^{2-3}$ o conhecimento relacionado aos fatores de risco e às formas de prevenção primária e secundária foi insuficiente entre os discentes participantes deste estudo. Fato preocupante, tendo em vista que essa temática é abordada durante a graduação e está inserida na prática dos profissionais da saúde, inclusive dos enfermeiros.

O conhecimento específico voltado ao HPV como um dos fatores de risco para o CCU foi expressivo entre os discentes, corroborando com dados de outro estudo. ${ }^{18}$ Entretanto, uma pesquisa realizada em uma universidade no interior da Etiópia, ${ }^{19}$ país com um dos menores Índice de Desenvolvimento Humano (IDH=0,442), ${ }^{20}$ demonstrou que 59,5\% dos discentes nunca ouviu falar em CCU, 79,4\% não compreendia as causas desse tipo de câncer e 37,7\% não sabia nenhum fator de risco.

Dessa forma, percebe-se que o conhecimento acerca do CCU e, consequentemente, dos fatores de risco, pode estar relacionado às oportunidades de contato com essa temática. 
Oportunidades dessa espécie devem ser dadas aos estudantes de graduação em Enfermagem, inclusive no âmbito prático.

Diferentemente da infecção pelo HPV, a relação entre o tabagismo como fator de risco para o CCU não foi estabelecida de modo consistente entre os participantes, estando em consonância com outros estudos que destacaram que os discentes não possuíam saber suficiente no que concerne à essa associação. ${ }^{21-22}$ Vale destacar que o tabagismo é comumente entendido como fator de risco para o desenvolvimento de outros tipos de cânceres, como nos casos do câncer de boca, ${ }^{23}$ o que pode ser justificado pelo baixo conhecimento sobre essa correlação.

A respeito da multiplicidade de parceria sexual e do início precoce da atividade sexual como fator de risco para o CCU, destaca-se um estudo realizado em Curitiba, cidade do Sul do Brasil, com média de acertos para a multiplicidade de parcerias sexuais de $91 \%$ e para o início precoce da vida sexual uma média de $63 \%$ entre os discentes de enfermagem estudados, ${ }^{24}$ sendo maior que os resultados encontrados no presente estudo. Observou-se, a partir da proporção de acertos, que o conhecimento acerca dos fatores de risco ainda é pautado na infecção pelo HPV, sendo necessário ações de intervenção e educação em saúde para esses discentes a fim de aprimorar o conhecimento referentes a esses aspectos.

Em relação à compreensão direcionada ao uso de preservativos como prevenção primária para o CCU, foi observada maior proporção entre os discentes que participaram de atividades extracurriculares, que estavam na segunda metade do curso e que assistiram à mulher com ênfase no CCU. Um estudo realizado com discentes ingressantes identificou que 51,7\% das entrevistadas não consideraram o preservativo como uma forma de prevenir o CCU e $84,9 \%$ não conheciam outras formas de prevenção. ${ }^{25}$

Percebe-se que o conhecimento dessa relação entre os discentes deste estudo ainda é insuficiente, tendo em vista que a média de acertos foi menor que 90\%. Assim, sugere-se aprimoramentos nas abordagens teóricas e práticas durante a graduação por meio de iniciativas 
como a inovação de tecnologias e as metodologias ativas de ensino, a fim de melhorar o conhecimento dos discentes.

Quanto à vacinação contra o HPV como forma de prevenção primária do CCU, notou-se associação estatística com a assistência à mulher com ênfase no CCU e expressiva proporção de acerto entre os discentes, corroborando com resultado de outro estudo. ${ }^{26}$ Acredita-se que a inclusão da imunização contra o HPV no Calendário Nacional de Vacinação em 2014 e a ampla divulgação por meio da mídia até os dias atuais pode ter contribuído para a popularização do conhecimento sobre o tema. ${ }^{27}$

Os resultados do que tange ao conhecimento voltado à prevenção secundária demonstraram lacunas consideráveis no processo de formação acadêmica. Observou-se que os discentes possuíam conhecimento insuficiente acerca do rastreio e da sintomatologia para o CCU, os quais são estratégias importantes para o diagnóstico precoce. ${ }^{4}$

O conhecimento insuficiente em relação a esse tipo de prevenção pode retardar a identificação dos sinais e sintomas do CCU, dificultando o diagnóstico e a adesão das mulheres às ações ofertadas pelos serviços de saúde. Nesse contexto, um estudo realizado em Uganda demonstrou que as mulheres desconheciam os sinais e sintomas do CCU, enfatizando a importância das ações de educação em saúde realizada pelos profissionais de saúde como um dos principais facilitadores para a triagem do CCU. ${ }^{28}$

A abordagem dos fatores de risco e da prevenção primária e secundária do CCU no âmbito acadêmico tem a finalidade de promover o desenvolvimento de habilidades em educação em saúde entre os discentes, almejando o aperfeiçoamento prático e profissional. ${ }^{29}$ Portanto, a falta de conhecimento vinculado a essa temática pode refletir negativamente no exercício profissional e implicar assistência com baixa resolutividade.

Nesse sentido, um estudo de revisão integrativa da literatura evidenciou que há insegurança entre os enfermeiros perante a temática e baixo investimento dos gestores em 
educação permanente, ${ }^{7}$ fragilizando as orientações acerca do CCU e o plano de ação para a prevenção do CCU na população feminina nos serviços de saúde.

Destacam-se como limitações deste estudo a inclusão apenas do conhecimento de discentes acerca da citada temática, desconsiderando a disposição e organização das aulas teóricas e práticas dadas pelos docentes da Instituição de Ensino Superior na qual o estudo foi desenvolvido. A partir da vivência docente ficaria mais propícia a identificação das lacunas existentes no processo de aprendizagem dos discentes e a constituição de novas estratégias de ensino, a fim de aproximar os discentes de discussões sobre os fatores de risco e prevenção do CCU.

\section{Conclusão}

Os resultados apontaram que as variáveis sociodemográficas e comportamentais entre os discentes não apresentaram correlação com o conhecimento referente aos fatores de risco e da prevenção para o CCU. Contudo, identificou-se associação do conhecimento acerca dessa temática com a participação em atividades extracurriculares, período do curso e assistência à mulher com ênfase no CCU durante a graduação.

Observou-se que considerar o tabagismo, a multiplicidade de parcerias sexuais e o início precoce da vida sexual como fator de risco para o CCU foi insuficiente entre o conhecimento dos participantes. Além disso, na perspectiva da prevenção secundária, rastreio e diagnóstico precoce, os resultados demonstraram lacunas consideráveis no conhecimento, sugerindo maior abordagem desses aspectos nas atividades teóricas e práticas durante a graduação em Enfermagem.

Tendo em vista que as associações encontradas estiveram relacionadas aos aspectos acadêmicos, destaca-se a relevância de fortalecer e aprimorar a abordagem dos fatores de risco e prevenção do CCU durante a graduação. Isso pode ser feito por meio da participação de discentes em atividades extracurriculares, além de outros caminhos teóricos e práticos. 
Para estudos futuros, vê-se a relevância de se utilizar a abordagem qualitativa com essa mesma temática, no intuito de ancorar os resultados baseados nos valores e percepções de cada discente de maneira específica e direcionada. Sugere-se a realização de estudos de intervenção com a finalidade de ampliar o escopo do conhecimento dos discentes pautado nos fatores de risco e a prevenção do CCU.

Os resultados deste estudo poderão ser usados como subsídio para discentes e docentes de cursos de enfermagem, bem como os enfermeiros, pois, com esse conhecimento, será possível compreender as nuances do processo de formação profissional e, assim, contribuir para atividades que abordem o CCU na graduação, influenciando positivamente a prática profissional.

\section{Referências}

1. Organização Pan-Americana da Saúde (OPAS). Controle integral do câncer do colo do útero: guia de práticas essenciais [Internet]. Washington (DC): OPAS; 2016 [cited 2020 Jan 20]. Available from: https://www.paho.org/hq/index.php?option=com_content\&view=article\&id=12813:controle-integral-docancer-do-colo-do-utero-guia-de-praticas-essenciais\&Itemid=40602\&lang=es

2. World Health Organization (WHO). Human papillomavirus (HPV) and cervical cancer [Internet]. 2019 [cited 2020 Jul 07]. Available from: https://www.who.int/news-room/fact-sheets/detail/humanpapillomavirus-(hpv)-and-cervical-cancer

3. Instituto Nacional de Câncer (INCA). Incidência estimada conforme a localização primária do tumor e sexo [Internet]. 2020 [acesso em 2020 jul 05]. Disponível em: https://www.inca.gov.br/numeros-de-cancer

4. Ministério da Saúde (BR), Secretaria de Atenção à Saúde, Departamento de Atenção Básica. Controle dos cânceres do colo do útero e da mama [Internet]. 2ª ed. Brasília (DF): Ministério da Saúde; 2013 [acesso em 2020 jan 20]. Disponível em: http://bvsms.saude.gov.br/bvs/publicacoes/controle_canceres_colo_utero_2013.pdf

5. Rosa RRPA. Redução da morbimortalidade por câncer de colo uterino. Rev Epidemiol Controle Infecç. 2016;6(3):131-7. doi: 10.17058/reci.v6i3.6633

6. Instituto Nacional de Câncer (INCA). Diretrizes brasileiras para o rastreamento do câncer do colo do útero [Internet]. 2a ed. Rio de Janeiro: INCA; 2016 [acesso em 2020 jan 20]. Disponível em: https://www.inca.gov.br/publicacoes/livros/diretrizes-brasileiras-para-o-rastreamento-do-cancer-do-colodo-utero 
7. Silva LR, Almeida CAPL, Sá GGM, Moura LKB, Araújo ETH. Educação em saúde como estratégia de prevenção do câncer do colo do útero: revisão integrativa. Rev Prev Infecç Saúde. 2017;3(4):35-45. doi: 10.26694/repis.v3i4.6708

8. Costa FKM, Weigert SP, Burci L, Nascimento KF. Os desafios do enfermeiro perante a prevenção do câncer do colo do útero. Rev Gest Saúde [Internet]. 2017 [acesso em 2019 out 21];17(Supl 1):55-62. Disponível em: http://www.herrero.com.br/files/revista/filef125a619c4b18a99efe6fdf22874fdd6.pdf

9. BRASIL. Ministério da Saúde. Portaria n 2.436, de 21 de setembro de 2017. Estabelece a revisão de diretrizes da Política Nacional de Atenção Básica (PNAB), no âmbito do Sistema Único de Saúde. Brasília, DF: Ministério da Saúde, 2017. Disponível em: https://bvsms.saude.gov.br/bvs/saudelegis/gm/2017/prt2436_22_09_2017.html. Acesso em: 21 out. 2019.

10. Maia RCB, Silveira BL, Carvalho MFA. Câncer do colo do útero: papel do enfermeiro na estratégia e saúde da família. Rev Cient FAEMA. 2018;9(1):348-72. doi: 10.31072/rcf.v9i1.517

11. AbdAllah AAA, Hummeida ME, Elmula IMF. Awareness and attitudes of nursing students towards prevention of cervical cancer. Cerv Cancer [Internet]. 2016 [cited 2020 Jul 07];1(1):1000106. Available from: https://www.omicsonline.org/open-access/awareness-and-attitudes-of-nursing-students-towardsprevention-of-cervical-cancer-ccoa-1000106.pdf

12. Dönmez S, Öztürk R, Kısa S, Weller BK, Zeyneloğlu S. Knowledge and perception of female nursing students about human papillomavirus (HPV), cervical cancer, and attitudes toward HPV vaccination. J Am Coll Health. 2019;67(5):410-7. doi: 10.1080/07448481.2018.1484364

13. Silva RGM, Nascimento VF, Santos POF, Ferreira MZJ. Teste de Papanicolaou: realização e conhecimento de acadêmicas de enfermagem. Rev Epidemiol Controle Infecç. 2019;9(1):81-6. doi: https://doi.org/10.17058/reci.v9i1.11592

14. Tonhom SFR, Moraes MAA, Pinheiro OL. Formação de enfermeiros centrada na prática profissional: percepção de estudantes e professores. Rev Gaúcha Enferm. 2016;37(4):e63782. doi: 10.1590/19831447.2016.04.63782

15. Kellogg C, Shu J, Arroyo A, Dinh NT, Wade N, Sanchez E, et al. A significant portion of college students are not aware of HPV disease and HPV vaccine recommendations. Hum Vaccin Immunother. 2019;15(7-8):1760-6. doi: 10.1080/21645515.2019.1627819

16. Pelullo CP, Esposito MR, Di Giuseppe G. Human papillomavirus infection and vaccination: knowledge and attitudes among nursing students in Italy. Int J Environ Res Public Health. 2019;16(10):1770. doi: 10.3390/ijerph16101770

17. Shetty S, Prabhu S, Shetty V, Shetty AK. Knowledge, attitudes and factors associated with acceptability of human papillomavirus vaccination among undergraduate medical, dental and nursing students in South India. Hum Vaccin Immunother. 2019;15(7-8):1656-65. doi: 
18. Burlamaqui JC, Cassanti AC, Borim GB, Damrose E, Villa LL, Silva L. Human papillomavirus and students in Brazil: an assessment of knowledge of a common infection - preliminary report. Braz J Otorhinolaryngol. 2017;83(2):120-5. doi: 10.1016/j.bjorl.2016.02.006

19. Mruts KB, Gebremariam TB. Knowledge and perception towards cervical cancer among female Debre Berhan University students. Asian Pac J Cancer Prev. 2018;19(7):1771-7. doi: 10.22034/APJCP.2018.19.7.1771

20. Programa das Nações Unidas para o Desenvolvimento (PNUD). Ranking IDH Global 2014. 2015 [cited 2020 Jul 07]. Available from: https://www.br.undp.org/content/brazil/pt/home/idh0/rankings/idhglobal.html

21. Lima CA, Amaral JG, Oliveira PP, Santos WJ, Rodrigues AB, Aguiar MIF. Cervical cancer: university students knoweledge. Rev Enferm UFPE On Line [Internet]. 2016 [cied 2020 Jul 07];10(8):2993-3003. Available from: https://periodicos.ufpe.br/revistas/revistaenfermagem/article/view/11369

22. Rachana KC, Giri R. Knowledge regarding cervical cancer among undergraduate female students at a selected college of Lalitpur, Nepal. Can Oncol Nurs J. 2019;29(3):184-8. doi: 10.5737/23688076293184188

23. Silva SR, Juliano Y, Novo NF, Weinfeld I. Comparative study of knowledge about oral cancer among undergraduate dental students. Einstein. 2016;14(3):338-45. doi: 10.1590/S1679-45082016AO3729

24. Okamoto CT, Faria AAB, Sater AC, Dissenha BV, Stasievski BS. Perfil do conhecimento de estudantes de uma universidade particular de Curitiba em relação ao HPV e sua prevenção. Rev Bras Educ Méd. 2016;40(4):611-20. doi: 10.1590/1981-52712015v40n4e00532015

25. Parreira BDM, Mendes LC, Canton HP, Gomes NS, Soares MBO, Silva SR. Knowledge, attitudes and university practices on prevention of cervical cancer. Rev UFPE On Line [Internet]. 2017 [cited 2020 Jul 07];11(Suppl 5):2116-21. Available from: https://periodicos.ufpe.br/revistas/revistaenfermagem/article/view/23366

26. Hino P, Freitas NC, Onofre PSC, Souza KL, Santos JO. Conhecimento de graduandos em enfermagem sobre a vacina contra o papilomavírus humano. Rev Rene. 2016;17(5): 586-92. doi: $10.15253 / 2175-6783.2016000500002$

27. Ministério da Saúde (BR), Secretaria de Vigilância em Saúde. Informe técnico sobre a vacina papilimavírus humano (HPV) na atenção básica [Internet]. Brasília (DF): Ministério da Saúde; 2014 [acesso em 2019 nov 14]. Disponível em: https://portalarquivos2.saude.gov.br/images/pdf/2015/junho/26/Informe-T--cnico-Introdu----o-vacinaHPV-18-2-2014.pdf

28. Ndejjo R, Mukama T, Kiguli J, Musoke D. Knowledge, facilitators and barriers to cervical cancer 
17 | Silva Júnior JA, Bezerra LLO, Freitas JLGS, Santos SMP, Queiroga RPF, Silva TRF

screening among women in Uganda: a qualitative study. BMJ Open. 2017;7(6):e016282. doi: 10.1136/bmjopen-2017-016282

29. Rocha JPJ, Oliveira KKD, Matoso LML, Dantas SLC, Maia CAAS. Conhecimento de acadêmicos sobre a prevenção do câncer de colo do útero e de mama. Rev Enferm UFSM. 2018;8(3):464-74. doi: $10.5902 / 2179769227839$

Editora Científica: Tânia Solange Bosi de Souza Magnago

Editora associada: Silvana Bastos Cogo

Financiamento: O presente trabalho foi realizado com os recursos próprios da equipe de pesquisa.

\section{Autor correspondente}

José Antonio da Silva Júnior

E-mail: joseantonio.030@hotmail.com

Endereço: Centro de Ciências Biológicas e da Saúde da Universidade Federal de Campina Grande - Av. Juvêncio Arruda, 795, Bodocongó, Campina Grande/PB

CEP: $58.109-790$

\section{Contribuições de Autoria}

\section{1- José Antonio da Silva Júnior}

Concepção da pesquisa, análise e interpretação dos dados e revisão final com participação crítica e intelectual no manuscrito.

\section{2- Luana Larissa Oliveira Bezerra}

Concepção da pesquisa, análise e interpretação dos dados e revisão final com participação crítica e intelectual no manuscrito.

\section{3- Javanna Lacerda Gomes da Silva Freitas}

Análise e interpretação dos dados e revisão final com participação crítica e intelectual no manuscrito.

\section{4- Sheila Milena Pessoa dos Santos}

Concepção da pesquisa, análise e interpretação dos dados e revisão final com participação crítica e intelectual no manuscrito.

\section{5- Rodrigo Pinheiro Fernandes de Queiroga}

Análise e interpretação dos dados. 
6- Trycia Ryane de Freitas Silva

Revisão final com participação crítica e intelectual no manuscrito.

\section{Como citar este artigo}

Silva Júnior JA, Bezerra LLO, Freitas JLGS, Santos SMP, Queiroga RPF, Silva TRF. O conhecimento dos discentes de enfermagem acerca do câncer de colo do útero. Rev. Enferm. UFSM. 2021 [Acesso em: Anos Mês Dia]; vol.11 e7: 1-18. DOI:https://doi.org/10.5902/2179769241938 